\title{
A public dataset of running biomechanics and the effects of running speed on lower extremity kinematics and kinetics
}

\author{
Reginaldo K Fukuchi ${ }^{\text {Corresp., }}{ }^{1}$, Claudiane A Fukuchi ${ }^{2}$, Marcos Duarte ${ }^{1,2}$ \\ 1 Biomedical Engineering Program, Universidade Federal do ABC, São Bernardo do Campo, São Paulo, Brazil \\ 2 Neuroscience and Cognition Graduate Program, Universidade Federal do ABC, São Bernardo do Campo, São Paulo, Brazil \\ Corresponding Author: Reginaldo K Fukuchi \\ Email address: regifukuchi@gmail.com
}

Background. The goals of this study were (1) to present the set of data evaluating running biomechanics (kinematics and kinetics), including data on running habits, demographics, and levels of muscle strength and flexibility made available at Figshare (DOI: $10.6084 / \mathrm{m}$ 9.figshare.4543435); and (2) to examine the effect of running speed on selected gait-biomechanics variables related to both running injuries and running economy. Methods. The lower-extremity kinematics and kinetics data of 28 regular runners were collected using a three-dimensional (3D) motion-capture system and an instrumented treadmill while the subjects ran at $2.5 \mathrm{~m} / \mathrm{s}, 3.5 \mathrm{~m} / \mathrm{s}$, and $4.5 \mathrm{~m} / \mathrm{s}$ wearing standard neutral shoes. Results. A dataset comprising raw and processed kinematics and kinetics signals pertaining to this experiment is available in various file formats. In addition, a file of metadata, including demographics, running characteristics, foot-strike patterns, and muscle strength and flexibility measurements is provided. Overall, there was an effect of running speed on most of the gait-biomechanics variables selected for this study. However, the foot-strike patterns were not affected by running speed. Discussion. Several applications of this dataset can be anticipated, including testing new methods of data reduction and variable selection; for educational purposes; and answering specific research questions. This last application was exemplified in the study's second objective. 


\section{A public dataset of running biomechanics and the effects of running}

\section{2 speed on lower extremity kinematics and kinetics}

4 Reginaldo Kisho Fukuchi ${ }^{1}$, Claudiane Arakaki Fukuchi ${ }^{2}$, Marcos Duarte ${ }^{1,2}$

5

$6 \quad{ }^{1}$ Biomedical Engineering Program, Universidade Federal do ABC, São Bernardo do Campo-SP,

$7 \quad$ Brazil

$8 \quad{ }^{2}$ Neuroscience and Cognition Graduate Program, Universidade Federal do ABC, São Bernardo

9 do Campo-SP, Brazil

11 Corresponding Author

12 Reginaldo Kisho Fukuchi ${ }^{1}$

13 Email address: regifukuchi@gmail.com 


\section{Abstract}

15 Background. The goals of this study were (1) to present the set of data evaluating running

16 biomechanics (kinematics and kinetics), including data on running habits, demographics, and

17 levels of muscle strength and flexibility made available at Figshare (DOI:

18 10.6084/m9.figshare.4543435); and (2) to examine the effect of running speed on selected gait-

19 biomechanics variables related to both running injuries and running economy. Methods. The

20 lower-extremity kinematics and kinetics data of 28 regular runners were collected using a three-

21 dimensional (3D) motion-capture system and an instrumented treadmill while the subjects ran at

$22 \quad 2.5 \mathrm{~m} / \mathrm{s}, 3.5 \mathrm{~m} / \mathrm{s}$, and $4.5 \mathrm{~m} / \mathrm{s}$ wearing standard neutral shoes. Results. A dataset comprising raw

23 and processed kinematics and kinetics signals pertaining to this experiment is available in

24 various file formats. In addition, a file of metadata, including demographics, running

25 characteristics, foot-strike patterns, and muscle strength and flexibility measurements is

26 provided. Overall, there was an effect of running speed on most of the gait-biomechanics

27 variables selected for this study. However, the foot-strike patterns were not affected by running

28 speed. Discussion. Several applications of this dataset can be anticipated, including testing new

29 methods of data reduction and variable selection; for educational purposes; and answering

30 specific research questions. This last application was exemplified in the study's second objective. 


\section{Introduction}

32

33

34

35

Long-distance running has become a very popular form of physical activity among individuals pursuing a healthy lifestyle (Stamatakis \& Chaudhury 2008). The health benefits of regular running are well known, however worrisome rates of running-related injuries have been reported and have associated burdens and economic costs (Hespanhol Junior et al. 2016).

Running biomechanics has been claimed to be associated with both running injury etiology (Hreljac 2004) and running economy (Moore 2016). Impact forces, foot pronation and shoes have all been linked to injuries although the literature is inconclusive about their role in the risk of running injuries (Nigg et al. 2015). Running foot strike patterns have also been the focus of great interest in the discussion pertaining biomechanical injury factors which has resulted in an increased number of studies examining their effects on the rate of injuries and on running biomechanics (Daoud et al. 2012; Hall et al. 2013). Another factor that has been related to running injuries is the excessive pace or excessive training volume (Nielsen et al. 2013). However, only a handful of studies have focused on examining the effect of running speed on gait biomechanics (Petersen et al. 2014; Schache et al. 2011), and the available evidence is rather conflicting. This can be partly explained by the fact that running biomechanics has been examined either without controlling the gait speed or by obtaining the data for a single controlled gait speed. In addition, although these studies added new data, they typically used small sample sizes and limited sets of biomechanical variables and considered only one part of the gait cycle (either the stance or swing phase), not to mention that the raw data from these studies are typically not freely available. Therefore, there is a need for studies that examine a larger set of runners across a range of gait speeds and that consider a larger set of biomechanical variables (e.g., kinematics and kinetics). 
Although a study including these features would greatly contribute to advancing

55 knowledge about the effect of gait speed, some challenges are likely to be encountered. The

56

57 58

59

60

61

62

complex, multivariate nature of biomechanics data challenges traditional data-analysis methods and, therefore, limits the ability of clinical-gait researchers to interpret these results and apply this knowledge to intervention procedures. To overcome these challenges and encourage the development of innovative tools that can address the nature of gait-biomechanics data, data sharing has been advocated (Ferber et al. 2016). Unfortunately, there are few publicly available datasets in the human movement science area (see, for example, Moore et al. 2015; Santos \& Duarte 2016). In fact, to our knowledge, there is no running biomechanics data sets with varying gait speeds available to the public. Therefore, a public data set of raw running biomechanics data would address this limitation and would welcome international research groups to use this data set to provide further insights about the related changes in biomechanics under varying running speed conditions. Therefore, the purposes of this study were (1) to present the set of raw and processed data on running biomechanics made available at Figshare (DOI:

10.6084/m9.figshare.4543435); and (2) to examine the effect of running speed on selected gaitbiomechanics variables associated with both injury etiology and running performance.

\section{Materials and Methods}

This study aimed to examine the effect of running speed on selected gait-biomechanics variables and to make the resulting dataset available in a public repository. The study was conducted at the Laboratory of Biomechanics and Motor Control (BMClab; http://demotu.org) at the Federal University of ABC (UFABC). The data collection was performed by experienced physiotherapist researchers. A pilot study with 5 subjects was conducted prior to the beginning 
77 the principal study. This study was approved by the local ethics committee of the UFABC

78 (CAAE: 53063315.7.0000.5594), and written, informed consent was obtained from each subject

79 prior to participation in the study. The data collection was designed to record the following

80 measurements, which are described in detail later: three-dimensional (3D) kinematics of the 2

81 lower limbs and pelvis, ground-reaction forces (GRF) during running on a treadmill at various

82 speeds, and the strength and flexibility of selected muscle groups and joints.

83

\section{$84 \quad 2.1 \quad$ Participants}

85 The study analyzed a convenience sample of 28 subjects who were recruited through 86 posted flyers, advertisement on the BMClab Internet home-page (http://demotu.org), and social

87 media. The inclusion criteria included being a regular runner with a weekly mileage greater than

$8820 \mathrm{~km}$, a minimum average running pace of $1 \mathrm{~km}$ in 5 minutes during $10-\mathrm{km}$ races, and

89 familiarity and comfort with running on a treadmill. The exclusion criteria were the presence of 90 any neurological or musculoskeletal disorder that compromises its locomotion or the use of any 91 assistive devices. The data related to demographics, running-training characteristics, previous 92 injuries, and other relevant information were made available in the public dataset (see also the 93 Table S1 of the supplementary material). 


\section{$95 \quad 2.2 \quad$ Equipment}

96 The running kinematics were collected via a 3D motion-capture system with 12 cameras

97 having $4 \mathrm{Mb}$ of resolution and the Cortex 6.0 software (Raptor-4, Motion Analysis, Santa Rosa,

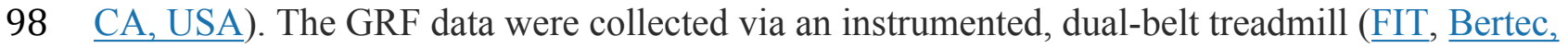

99 Columbus, OH, USA).

100 The cameras were distributed around the laboratory such that they aimed at the

101 instrumented treadmill's motion-capture volume (Figure 1). The cameras were mounted in a

102 metallic truss setup structure with a length of $11.5 \mathrm{~m}$, a width of $9.3 \mathrm{~m}$, and a height of $2.8 \mathrm{~m}$.

103 This structure allowed positioning some cameras with varying elevations; however, most were

104 placed atop the truss setup to optimize capturing the markers during the running trials (Figure 1).

105 The instrumented treadmill was mounted over a pit, with the treadmill surface at the same level

106 as the laboratory floor (Figure 1).

107

108

$* * * * *$ Insert Figure 1 near here $* * * * *$

109

110

The Cortex 6.0 software (Motion Analysis, Santa Rosa, CA, USA) was used to (1)

111

calibrate the motion-capture volume; (2) capture and identify the reflective markers; and (3)

112 prepare the data and export it to the $\mathrm{c} 3 \mathrm{~d}$ file format. To provide an unbiased, raw dataset having

113 marker trajectories and force signals, no further processing (e.g., filtering, gap filling) was

114 performed on the data.

115 The motion-capture volume consisted of an area $3.1 \mathrm{~m}$ long, $2.3 \mathrm{~m}$ wide, and $1.2 \mathrm{~m}$ high,

116 and this volume was calibrated daily. The system was deemed properly calibrated only if the

117 length of the calibration wand, which was measured by the capture system, was within $0.10 \mathrm{~mm}$ 
118 of the true wand length. The rates of acquisition of the kinematics and kinetics data were set at

$119150 \mathrm{~Hz}$ and $300 \mathrm{~Hz}$, respectively.

120 The laboratory-coordinate system used for the study was the same as that proposed by the

121 International Society of Biomechanics (Wu \& Cavanagh 1995) and, as shown in Figure 1,

122 contained the following.

123 - X-axis in the direction of gait progression and positive pointing forward

$124 \quad$ Y - -axis in the vertical direction and positive pointing upward

125 - Z-axis in the medial-lateral direction and positive pointing to the right

126 To record the strength and flexibility measures of selected muscle groups and joints, a

127 hand-held dynamometer (HHD) (range: 0-1330 N; accuracy: $\pm 1 \%$; resolution: $1 \mathrm{~N}$; Nicholas

128 MMT, Lafayette Instruments, Lafayette, IN, USA) and a magnetic-angle locator (Model 700;

129 Johnson Level \& Tool Mfg. Co., Inc., Mequon, WI, USA) were used, respectively.

130

\section{$\begin{array}{lll}131 & 2.3 & \text { Protocol }\end{array}$}

132 The data-collection protocol involved the following procedures.

133 1. Interview. Upon arrival, the participant was introduced to the laboratory and given a brief

134 explanation of the experimental procedures. Then, the participant was asked to provide

135 written informed consent and undergo a brief interview regarding eligibility criteria,

136 demographic data, and running habits.

137 2. Preliminary measurements. Body height and mass were measured, and shoe-fitting was

138

139 conducted to determine the appropriate shoe size. All participants wore neutral laboratory-controlled shoes (Nike Dual Fusion X). 
140 3. Marker placement. The study used 48 technical and anatomical reflective markers (see

141

142

143

144

145

146

147

148

149

150

151

152

153

154

155

156

157

158

159

160

161

162 details in Table 1 and Figure 2). Clusters with 4 technical markers, placed in a rigid shell, were used on the thigh and shank segments. Their design was based on Cappozzo et al. (1997). These shells were securely fastened to the segments using a combination of elastic and Velcro straps.

4. Standing calibration trial. A template was used to align the subject's feet in a standardized position such that the long axes of the feet were parallel to the X-axis of the laboratory-coordinate system (Figure 2). Then, the markers' 3D coordinates were recorded for $1 \mathrm{~s}$.

5. Removal. After the calibration trial, the anatomical markers were removed except for those considered both anatomical and technical markers (T/A in Table 1).

6. The force plates were zeroed, the subject was asked to step onto the treadmill, and the following protocol was followed.

a. The subject walked at $1.2 \mathrm{~m} / \mathrm{s}$ for $1 \mathrm{~min}$ to become familiar with the treadmill.

b. Next, the subject was asked to stay on the left belt of the treadmill, the belt speed was incrementally increased to $2.5 \mathrm{~m} / \mathrm{s}$, and after a 3-min accommodation period at this velocity, the data were recorded for $30 \mathrm{~s}$. This procedure was repeated at speeds of $3.5 \mathrm{~m} / \mathrm{s}$ and $4.5 \mathrm{~m} / \mathrm{s}$, always in the same sequence.

c. After the running trials, the treadmill speed was again set to $1.2 \mathrm{~m} / \mathrm{s}$ for a $1-\mathrm{min}$ cool-down period prior to being stopped.

7. Measuring the flexibility of the iliotibial band using the angle locator during the Ober's test procedure. In brief, the test is performed with the subject lying on his/her side. The examiner then passively move the tested leg (leg on top) into hip flexion, abduction, and 
extension and lowers the limb into adduction until it stops limited by soft tissue stiffness.

164 Further details about this test procedure can be found in Fukuchi et al. (2014).

165 8. Measuring the flexibility of the hip flexors using the angle locator during the Thomas' to take these measurements were described previously (Fukuchi et al. 2014).

174

\section{$* * * * *$ Insert Table 1 near here $* * * * *$}

176 anatomical-frame conventions proposed previously (Cappozzo et al. 1995; Fukuchi et al. 2014).

182 A model template file (RBDSmodelV3D.mdh) for the Visual 3D software (C-Motion Inc., 183 Germantown, MD, USA) is available at Figshare. This .mdh file is an ASCII file containing the 184 definitions of all landmarks, segments, and segment properties adopted by the present study. 


\section{$186 \quad 2.4 \quad$ Data processing and analysis}

187

Raw marker-trajectory data and GRF data were filtered using a fourth-order, low-pass

188 Butterworth filter with the same cut-off frequency of $10 \mathrm{~Hz}$ (Kristianslund et al. 2012). The foot

189 strike and toe off were determined when the vertical GRF crossed a $20-\mathrm{N}$ threshold level. The

190 foot strike patterns were determined using the strike index, which was calculated as the ratio of

191 the center of pressure (COP) position in relation to the heel position, at foot strike, and the length

192 of the foot. The measurements were taken, however, during instrumented treadmill running

193 instead of on an overground condition as originally proposed by Cavanagh \& Lafortune (1980).

194 In addition, 3D hip, knee, and ankle angles were calculated using Cardan angles, with the

195 distal segment expressed relative to the proximal segment and adopting the following

196 convention: the first rotation described occurred in the medial-lateral axis (Z-axis, perpendicular

197 to the sagittal plane), which defines the flexion-extension movement; the third rotation described

198 was around the longitudinal axis (Y-axis, perpendicular to the transverse plane), which defines

199 the internal/external rotations; and the second rotation described was around an axis

200 perpendicular to the previous two axes, which in the anatomic position represents the anterior-

201 posterior axis (X-axis, perpendicular to the frontal plane), where abduction/adduction occurs.

202 This convention is defined simply as the Z-X-Y convention and is frequently used to describe

203 lower extremity rotations (Cappozzo et al. 1995). The net internal joint torques were represented

204 in the joint-coordinate system (Schache \& Baker 2007) and were calculated using a standard

205 inverse-dynamics approach. Joint powers were calculated as the scalar product of joint torques

206 and joint angular velocities. The joint kinetic and the GRF variables were normalized by the

207 subject's body mass. 
101 time points. Then, these curves were averaged across trials, resulting in one curve comprising each subject's average pattern. The number of footfalls varied with subject and

211 speed, but the minimum number was always greater than 30 . Next, to enable statistical

212 comparison, discrete variables were calculated for each curve. Global maximum and minimum

213 values for the joint angles and joint torques curves, GRF impulses, and joint work were

214 considered for further analysis. The GRF impulses and joint work were calculated as the area

215 under the GRF-time and joint power-time curves, respectively. The stride length and cadence

216 were also calculated as the spatiotemporal gait parameters. These variables have been examined

217 in previous studies related to running biomechanics in the context of injury etiology, running

218 performance and aging (Fukuchi \& Duarte 2008; Fukuchi et al. 2011; Fukuchi et al. 2014;

219 Fukuchi et al. 2016; Hall et al. 2013). The Visual 3D software program (C-motion Inc.,

220 Germantown, MD, USA) was used to filter the marker and GRF data and to calculate joint

221 kinematics and kinetics. Finally, these time-normalized data were exported as ASCII text files.

222 Then, the discrete variables, GRF impulses, and joint work were calculated using in-house

223 algorithms written in Matlab 9.0 2016a (Mathworks Inc., Natick, MA, USA). A file written for

224 the Visual 3D software program (RBDSpipelineV3D.v3s) is available at Figshare. This file is in

225 text format and contains a series of pipeline Visual 3D commands that were used to process the

226 raw c3d files, which are also available at Figshare. In addition to the raw c3d files, the time-

227 normalized kinematic and kinetic data for each subject are available as ASCII files at Figshare

228 (see the Results section for details). An exemplary Matlab code is also available in the

229 supplementary material. 


\section{$231 \quad 2.5 \quad$ Statistical analysis of the processed data}

232 The normality and homogeneity of variances assumptions of the dependent variables were

233 tested using the Bartlett's test. Either one-way ANOVAs or Kruskal-Wallis tests were conducted

234 to examine the effect of running speed on gait-biomechanics variables when the dependent

235 variables did or did not address the assumptions, respectively, at a significance level of 0.05.

236 Whenever a main effect was observed, post-hoc analysis was conducted using t-tests with

237 Bonferroni adjustments to minimize type I statistical errors. A multinomial logistic regression

238 analysis was performed to determine the effect of running speed (the predictor) on foot-strike

239 patterns (the categorical response variable). The statistical calculations were performed in Matlab

$2409.02016 \mathrm{a}$ (Mathworks Inc., Natick, MA, USA) using the Statistics toolbox.

241

\section{Results}

243 Both the raw and processed data and a metadata file are available at Figshare (DOI:

244 10.6084/m9.figshare.4543435); the data is stored in ASCII (text) format with tab-separated

245 columns that can be downloaded as a single compressed file that is made available under the CC-

246 BY license (https://creativecommons.org/licenses/by/4.0/).

\section{$248 \quad 3.1 \quad$ Raw data}

249 The raw data are stored in both $\mathrm{c} 3 \mathrm{~d}$ and text file formats. The $\mathrm{c} 3 \mathrm{~d}$ file format is a flexible

250 binary file containing all the unprocessed data from a captured trial. This file format is supported

251 by the main motion-capture manufacturers and other biomechanics software programs (e.g.,

252 Visual 3D). Although the Cortex software program, which was used to collect data, does offer

253 the capability of processing and analyzing data, the raw files available in the present dataset 
254 contain only 3D, raw marker coordinates and transformed force signals (i.e. forces $(\mathrm{N})$

255 transformed into the laboratory coordinate system).

256 The c3d files contain both the raw marker trajectories and force signals (both raw analog

257 signals $(\mathrm{V})$ and transformed signals $(\mathrm{N})$ ) in a single file. In contrast, separate text files were

258 created for markers and forces signals, as these signals were sampled at $150 \mathrm{~Hz}$ for kinematics

259 and $300 \mathrm{~Hz}$ for kinetics data. In addition, there is one c3d and one ASCII text file containing

260 only marker trajectories corresponding to one second of the standing, anatomical calibration data

261 of each subject. Finally, the average time-normalized kinematics (joint angles) and kinetics (joint

262 torques, joint powers, and GRF) data for each subject are available in the ASCII file. Hence, as

263 the running trials were performed on an instrumented treadmill at three distinct gait speeds $(2.5$

$264 \mathrm{~m} / \mathrm{s}, 3.5 \mathrm{~m} / \mathrm{s}$, and $4.5 \mathrm{~m} / \mathrm{s}$ ), there are four c3d files and eight text files per subject. Table 2

265 describes the file-naming convention used for the raw dataset.

266

267

$* * * * *$ Insert Table 2 near here $* * * * *$

268

269

The abbreviation RBDS in the file names stands for "Running Biomechanics Dataset"

270

and $\mathrm{xxx}$ refers to the subject's identification number (e.g., the first subject is 001). The c3d files

271 can be analyzed using the Visual 3D software program and the supplied model template file

272 (.mdh) along with the pipeline command files (.v3s) or other software, including Mokka

273 (http://biomechanical-toolkit.github.io/mokka/). The ASCII files with marker trajectories contain

27497 columns, with the first column containing the recording time (in seconds) and the remaining

27596 columns being the $\mathrm{X}, \mathrm{Y}$, and $\mathrm{Z}$ coordinates (in millimeters) of the markers in the laboratory-

276 coordinate system, as described in Table 3. The number of columns varied between the static 
277 and running trials (145 vs. 97, respectively) since the markers deemed solely anatomical were

278 removed before the running trials (see the Methods section). The headers of the marker files

279 contain the markers' labels (except for the first column, which is elapsed time) and are consistent

280 with the "label" column in Table 1. In turn, the columns of the forces ASCII files comprise the

281 forces, center of pressure, and free moment about the vertical axis measured by the instrumented

282 treadmill and transformed on to the laboratory-coordinate system. Each force file has the

283 following header: Time [s], Fx [N], Fy [N], Fz [N], COPx [mm], COPy [mm], COPz [mm], and

$284 \mathrm{Ty}[\mathrm{Nm}]$, followed by data in 9000 rows and 8 columns with 6-digit numerical precision.

285

286

287

\subsection{Metadata}

One file named RBDSinfo (in both .txt and .xlsx formats) is supported with metadata, demographics, running characteristics, foot-strike patterns, and muscle-strength and flexibility measurements for each subject. Below is the coding for the metadata. The first word identifies 290 the name of the column in the header.

291 1. Subject: number of subjects (from 1 to 28 ).

292 2. Filename: names of files, including format extensions. Table 2 provides descriptions of 293 the files.

294 3. Age: subject's age in years.

295 4. Height: subject's height in centimeters, measured with a calibrated stadiometer.

296 5. Mass: subject's body mass in kilograms, measured with a calibrated scale.

297 6. Gender: subject's gender (M or F).

298 7. Dominance: answer to the question "What leg would you use if you had to kick a ball (R 299 or L)?” . 
300 8. Level: answer to multiple-choice question about self-assessed level of running

301 performance (only recreational; recreational competitive; competitive; elite).

302 9. Experience: number of months of regular running practice (at least 3 times/week).

303 10. SessionsPerWk: number of running training sessions per week.

304 11. Treadmill: number of treadmill running training sessions per week.

305 12. Asphalt: number of running training sessions on an asphalt surface per week.

306 13. Grass: number of running training sessions on a grass surface per week.

307 14. Trail: number of trail running training sessions per week.

308 15. Sand: number of running training sessions on sand per week.

309 16. Concrete: number of running training sessions on a concrete surface per week.

310 17. SurfaceAlt: number of running training sessions per week on other surfaces not listed

311 previously.

312 18. RunGrp: whether the subjects participated in a running training group, as self-declared 313 (Yes or No).

314 19. Volume: weekly running training volume (kilometers/week).

315 20. Pace: average running pace in the shortest long-distance running race (minutes/ $316 \quad$ kilometer).

317 21. RaceDist: running race distance participated in recently, as self-declared (in kilometers).

318 22. Injury: answer to the question "Have you experienced any injury or pain that has 319 significantly affected your running practice?” (Yes or No).

320 23. InjuryLoc: anatomical location of the most recent injury.

321 24. DiagnosticMed: answer to the question "Was this injury medically diagnosed?" (Yes or 322 No). 
323 25. Diagnostic: diagnosis of running-related injury, as self-declared.

324 26. InjuryOnDate: approximate date of onset of injury symptoms, as self-declared

$325 \quad(\mathrm{dd} / \mathrm{mm} /$ yyyy $)$.

326 27. ShoeSize: size of running shoes, as self-declared (US standard).

327 28. ShoeBrand: preferred running shoe manufacturer, as self-declared.

328 29. ShoeModel: model of running shoes, as self-declared.

329 30. ShoePairs: current number of pairs of running shoes, as self-declared.

330 31. ShoeChange: answer to the following multiple choice question "How often do you

331 replace your running shoes?" (less than 6 months; between 7 months and 1 year; between

3321 and 2 years; more than 2 years).

333 32. ShoeComfort: comfort rating of their current running shoes, as self-declared on a 10-

334 point rating scale. An average rating score was calculated if they had more than one pair 335 of shoes.

336 33. ShoeInsert: type of foot insert (if any) worn in their running shoes (off-the-shelf insoles; 337 orthotics; taping; none).

338 34. RFSI25: right foot-strike pattern (rearfoot, midfoot, or forefoot) while running at $2.5 \mathrm{~m} / \mathrm{s}$ 339 (see description in Methods).

340 35. RFSI35: right foot-strike pattern (rearfoot, midfoot, or forefoot) while running at $3.5 \mathrm{~m} / \mathrm{s}$ $341 \quad$ (see description in Methods).

342 36. RFSI45: right foot-strike pattern (rearfoot, midfoot, or forefoot) while running at $4.5 \mathrm{~m} / \mathrm{s}$ 343 (see description in Methods).

344 37. LFSI25: left foot-strike pattern (rearfoot, midfoot, or forefoot) while running at $2.5 \mathrm{~m} / \mathrm{s}$ 345 (see description in Methods). 
346 38. LFSI35: left foot-strike pattern (rearfoot, midfoot, or forefoot) while running at $3.5 \mathrm{~m} / \mathrm{s}$ $347 \quad$ (see description in Methods).

348 39. LFSI45: left foot-strike pattern (rearfoot, midfoot, or forefoot) while running at $4.5 \mathrm{~m} / \mathrm{s}$ 349 (see description in Methods).

350 40. RThomas: angle of the right thigh relative to the horizontal during the Thomas' test measured with a magnetic angle locator, in degrees (see description in Methods). Positive and negative values represent the thigh below and above a line parallel to the therapeutic bench.

41. LThomas: angle of the left thigh relative to the horizontal during the Thomas' test measured with a magnetic angle locator, in degrees (see description in Methods). Positive and negative values represent the thigh below and above a line parallel to the therapeutic bench.

42. ROber: angle of the right thigh relative to the horizontal during the Ober's test measured with a magnetic angle locator, in degrees (see description in Methods). Positive and negative values represent the thigh below and above a line parallel to the therapeutic bench.

43. LOber: angle of the left thigh relative to the horizontal during the Ober's test measured with a magnetic angle locator, in degrees (see description in Methods). Positive and negative values represent the thigh below and above a line parallel to the therapeutic bench.

44. RHIPABD: average maximal isometric voluntary contraction (MIVC) of the right hip abductors measured by a hand-held dynamometer (HHD) in kilograms (see Fukuchi et al. 2014). 
45. LHIPABD: MIVC of the left hip abductors measured by an HHD in kilograms.

46. RHIPEXT: MIVC of the right hip extensors measured by an HHD in kilograms.

47. LHIPEXT: MIVC of the left hip extensors measured by an HHD in kilograms.

372

48. RHIPER: MIVC of the right hip external rotators measured by an HHD in kilograms.

49. LHIPER: MIVC of the left hip external rotators measured by an HHD in kilograms.

50. RHIPIR: MIVC of the right hip internal rotators measured by an HHD in kilograms.

51. LHIPIR: MIVC of the left hip internal rotators measured by an HHD in kilograms.

376

377

378

379

380

381

382

383

384

385

386

387

388

389

390

391

\subsection{Processed data}

The processed data comprise average 3D time-normalized joint angles (hip, knee, and ankle), joint torques, and GRFs along with the joint powers at the sagittal plane. These data have six-digit precision (except the percentage column, which is an integer number), and they are stored in a single tab-separated ASCII text file. The processed data were stored such that their columns consisted of the following variables: Gait cycle (101 x 1) [percentage], 3D joint angles (101 x 9) [degrees], 3D joint moments (101 x 9) [Nm/kg], 3D GRF (101 x 3) [N/kg], and scalar joint powers $(101 \times 3)[\mathrm{W} / \mathrm{kg}]$. The numbers within parentheses represent the dimensions of the matrix of data (number of rows and columns), considering only one gait speed and one lower limb. Table 3 displays the arrangement of the first 25 columns of processed data stored in the ASCII files. Since there are three gait speeds $(2.5 \mathrm{~m} / \mathrm{s}, 3.5 \mathrm{~m} / \mathrm{s}$, and $4.5 \mathrm{~m} / \mathrm{s})$ and two lower limbs (right and left), the resultant matrix has 101 rows and 145 columns (144 columns of biomechanics data plus one column of gait-cycle percentage data). 


\section{$393 \quad 3.4 \quad$ Effect of gait speed on running biomechanics}

394 To study the effects of gait speed on running biomechanics, we compared the kinematics 395 and kinetics running patterns of the subjects across gait speeds. Note that the subjects had an 396 accommodation period at each running speed (see Protocol in the Methods section) before the 397 data were recorded. Figures $\mathbf{3}$ and $\mathbf{4}$ show the average pattern (across subjects) of the lower398 extremity 3D joint angles and the joint torques curves, respectively. Figure 5 shows the average 399 GRF curves in the medial-lateral, anterior-posterior, and vertical directions along with the lower 400 extremity joint power curves at the sagittal plane. Overall, an increase could be observed in the 401 magnitude of both kinematics (joint angles) and kinetic (torques, GRFs, and powers) variables 402 following an increase in gait speed.

403 For a more specific, quantitative examination of the effects of gait speed, Table 4 shows 404 the results of the descriptive and inferential statistical analyses. Six of the 24 variables did not 405 meet the assumptions for ANOVA and they were compared using Kruskal-Wallis tests. Figure 6 406 shows repeated-measures plots with the distribution of the subjects' data across running speeds 407 for all variables that had significant effects. The results of the post-hoc analyses are indicated 408 whenever a significant difference was found in the pairwise comparisons. The running-speed 409 conditions of $2.5 \mathrm{~m} / \mathrm{s}, 3.5 \mathrm{~m} / \mathrm{s}$, and $4.5 \mathrm{~m} / \mathrm{s}$ are defined hereafter as $\mathrm{V} 1, \mathrm{~V} 2$, and V3, respectively. 


\section{3.4.1 Gait kinematics}

412 A main effect of speed was observed for both stride length and cadence but not for stride

413 width (Table 4). The post-hoc analyses revealed that both stride length and cadence increased

414 significantly for all conditions tested (Figure 6 and Table 4).

415 Overall, the lower extremity joint angles were affected by running speed, since main

416 effects were observed in the peak angles of the hip, knee, and ankle joints, except for ankle

417 dorsiflexion (Figure 3 and Table 4). The maximal angles of hip extension, hip flexion, and knee

418 flexion differed across all possible comparisons. Compared to V1, the relative increases of these

419 variables at V2 and V3 were, respectively, in degrees: hip extension (4.4, 8.2), hip flexion (9.0,

420 16.8), and knee flexion $(15.1,25.6)$. In contrast, when V2 and V3 were compared with each

421 other, the peak ankle plantar flexion was not altered. The maximum ankle eversion angle also

422 exhibited higher values at higher speeds; however, the post-hoc analysis revealed that this

423 variable differed only when V1 and V3 were compared.

424 The foot-strike pattern distribution in V1, V2, and V3 were, respectively, rearfoot strikers

425 (RFS): 68\%, 68\%, 61\%; midfoot strikers (MFS): 14\%, 18\%, 21\%; and forefoot strikers (FFS):

$42618 \%, 14 \%, 18 \%$. Contrary to our hypothesis, the coefficients of the multinomial logistic

427 regression model revealed that foot-strike patterns were not affected by gait speed. The

428 probability of changing an RFS pattern (reference category) to either an FFS pattern $\left(\beta_{0}=-1.56 \pm\right.$

$\left.4291.32 ; \mathrm{IC}_{0}[-4.14 ; 1.02] ; \mathrm{p}_{0}=0.236 ; \beta_{1}=0.05 \pm 0.37 ; \mathrm{IC}_{1}[-0.66 ; 0.77] ; \mathrm{p}_{1}=0.882\right)$ or an MFS

430 pattern $\left(\beta_{0}=-2.21 \pm 1.33 ; \mathrm{IC}_{0}[-0.45 ; 0.96] ; \mathrm{p}_{0}=0.477 ; \beta_{1}=0.27 \pm 0.36 ; \mathrm{IC}_{1}[-0.45 ; 0.96] ; \mathrm{p}_{1}=\right.$

$4310.477)$ remained unaltered by any increment in gait speed. The term $\beta_{0} \pm$ se includes the

432 coefficient and standard error (se) of the constant; $\beta_{1} \pm$ se includes the coefficient and se of the 
433 predictor (gait speed); IC is the confidence interval of the coefficients; and $\mathrm{p}$ is the associated $\mathrm{p}$ 434 value.

435

\section{$436 \quad 3.4 .2 \quad$ Gait kinetics}

Overall, there was an effect of running speed on joint torques (in $\mathrm{Nm} / \mathrm{kg}$ ), joint work (in $\mathrm{J} / \mathrm{kg}$ ), and GRF variables (in Ns/kg), as can be seen in Figure 4, Figure 5, and Figure 6 and

Table 4. Compared to V1, the percentage of increase in hip extensor and flexor torque peaks at higher running speeds (V2 and V3), compared to V1, were 0.31 and 0.61 ; and 0.37 and 0.70 ,

441 respectively. In addition, compared to V1, at V2 and V3, the knee extensor torque increased 0.34

442 and 0.57 , respectively, and the ankle extensor torque increased 0.21 and 0.31 , respectively. In 443 contrast, no difference was found when V2 and V3 were compared to each other. In addition, a 444 main effect of running speed was found at the ankle flexor torque but only when V1 and V3 were 445 compared. Contrary to our hypothesis, the knee abduction impulse (area under the torque-time 446 curve) was not affected by running speed (Table 4). Compared to V1, the GRF propulsive, GRF

447 braking, and GRF vertical impulses increased $0.07,0.15,0.08$, respectively, at V2 and $0.13,0.22$, 448 and 0.15 , respectively, at V3 (Table 4). The post-hoc analyses found effects for all conditions 449 tested in the aforementioned variables, except for the GRF braking impulses between V2 and V3. 450 The hip and ankle positive works were affected in all tested conditions. Compared to V1, they 451 were increased 0.69 and 0.14 , respectively, at V2 and 1.62 and 0.32 , respectively, at V3. In 452 contrast, the knee positive work remained constant when V2 and V3 were compared to each 453 other. However, compared to V1, it increased 0.17 and 0.23 , respectively, at V2 and V3.

454 Compared to V1, the hip, knee, and ankle negative joint work increased 0.16, 0.65, and 0.19, 455 respectively, at V2 and $0.39,1.53$, and 0.39 , respectively, at V3 (Table 4). The distribution of 
456 positive and negative work across lower extremity joint are shown in Figure S1 of the

457 supplementary material.

458

459

$* * * * *$ Insert Figure 3 near here $* * * * *$

460

461

$* * * * *$ Insert Figure 4 near here $* * * * *$

462

463

$* * * * *$ Insert Figure 5 near here $* * * * *$

464

465

$* * * * *$ Insert Figure 6 near here $* * * * *$

466

467

$* * * * *$ Insert Table 4 near here $* * * * *$

468 


\section{Discussion}

470 This study presented a publicly available dataset on regular runners' gait biomechanics

471 (kinematics and kinetics), demographics, running habits, muscle strength, flexibility, and foot-

472 strike patterns. Biomechanical datasets have begun to be accessible in public repositories

473 recently (see Moore et al. 2015; Santos \& Duarte 2016, and the references therein). However,

474 these datasets primarily consist of walking and posture data. We provided both raw data (in two

475 formats: .c3d and .txt) and processed data for reuse, along with metadata and other files that can

476 be used to reproduce the processed data. In addition, the study examined the effect of running

477 speed on selected gait variables commonly associated with running injuries and running

478 economy. The study observed that running speed significantly affected lower-extremity

479 kinematics and kinetics.

480 Even though there has been an increased number of publications about running

481 biomechanics, there is a scarcity of publicly available running data sets which hampers the

482 dissemination of biomechanics data and prevents a wider use of published data. To help address

483 this problem, we presented a data set of running biomechanics of regular runners. Compared to

484 other available gait data sets, the present set include both raw and processed data in various files

485 formats. In addition, running biomechanics at different controlled gait speeds, from multiple gait

486 cycles, considering both limbs and both kinematics and kinetics data are provided. Furthermore,

487 a metadata file is included with the necessary information pertaining each file and participant of

488 the study to enhance the dissemination and wide use of the data. There are certainly other data

489 sets previously published that fulfil the same recommendations desired to disseminate and

490 enhance the reuse of data, however, to our knowledge, none of them assessed running

491 biomechanics (Moore et al. 2015). 
493 greater stride frequency. The stride length increased to a greater extent than stride frequency

494 (39.3\% vs. 9.3\% on average across the gait speeds). Dorn et al. (2012) reported similar results, in

495 which the stride length increased at higher rates at long-distance running speeds on the ground.

496 Schache (2014) stated that the results of Dorn et al. suggest that to increase their running speed,

497 humans choose to push the ground more forcefully rather than more frequently, particularly, at

498 slow-to-medium pace running. This is also compatible with the higher values we found for the

499 GRF horizontal propulsive impulse. The peak flexion and extension values of the hip, knee, and

500 ankle angles also increased at higher running speeds, except for the peak ankle dorsiflexion

501 angle. These results were expected, since the runners had to use larger strides, and thus greater

502 joint displacement, to cope with higher running speeds. Similar results have been reported

503 elsewhere (Dorn et al. 2012). The present study observed no change in the foot-strike patterns as

504 running speeds increased. Although there is a general understanding that the point of contact

505 shifts from the rear toward the anterior part of the foot as running speed increases (Cheung et al.

506 2016), this may not be true for speeds below $5 \mathrm{~m} / \mathrm{s}$ (Breine et al. 2014; Hatala et al. 2013), i.e.

507 within the range adopted in the present study. This contrasting evidence across studies highlights

508 the fact that the relationship between running speed and foot-strike patterns is complex and needs

509 to be examined further, particularly considering long distance running pace. Several factors may

510 explain the differences in the findings across studies such as the range of running speeds, running

511 surface (treadmill vs. overground), shoes, and different equipment or measurement methods used

512 to quantify foot-strike patterns. Therefore, these factors need to be considered in future studies.

513 Foot eversion (pronation) has long been associated with running injuries; however, there is

514 limited understanding of whether this is influenced by running speed. Although the present study 
515 observed a significant main effect of running speed on the peak ankle eversion angle, the post-

$516 h o c$ analysis revealed differences only when speeds of $2.5 \mathrm{~m} / \mathrm{s}$ and $3.5 \mathrm{~m} / \mathrm{s}$ were compared.

517 Similar results have been reported in recreational runners during treadmill running at

518 comfortable speeds (Munoz-Jimenez et al. 2015).

519 In general, the lower extremity joint torques and joint work were also affected by

520 increased running speed. In particular, the hip torques (both flexion and extension), hip work

521 (both positive and negative), and ankle positive work were all significantly affected by running

522 speed in all conditions tested. The important contribution of the ankle plantar flexors to

523 generating propulsive force and thereby increasing gait speed has been investigated both

524 experimentally and through simulation studies (Hamner \& Delp 2013; Schache et al. 2014).

525 Regarding hip-joint loading, there is evidence that the participation of the hip in power

526 generation increases non-linearly as a function of running speed (Schache et al. 2011). Similar

527 behavior was observed in the present study when the rate of increase in hip power generation

528 was not constant compared to the work and torque at the knee and ankle (see relative increase in

529 Table 4). This finding may be explained by the fact that the work done by the hip muscles to

530 accelerate the leg during the swing phase increases at a faster rate to move the leg forward more

531 rapidly. The knee extension torque and positive work were also affected by running speed, but to

532 a lesser extent than the hip and ankle, since they remained unaltered when speeds of $3.5 \mathrm{~m} / \mathrm{s} v s$.

$5334.5 \mathrm{~m} / \mathrm{s}$ were compared. In line with our hypothesis, the GRF horizontal and vertical impulses

534 were affected by running speed. These results were expected, since the leg must apply higher

535 impulses to the surface to increase gait speed. In particular, the increment of the GRF vertical

536 impulse with increased speed was only about $2.5 \%$ on average, although it was statistically

537 significant. 
539 in previous studies, including failing to consider both the stance and swing phases of the gait

540 cycle, small sample sizes, limited joints and set of variables (e.g., only kinematics or kinetics);

541 however, other limitations persist. The use of discrete variables from time-series curves may be

542 too simplistic to deal with the complex nature of gait-biomechanics data (Lai et al. 2009). Even

543 the area under the force-time and power-time curves may not be sufficient to capture the overall

544 pattern of the subjects. While our results seem to be in agreement with those of a handful of

545 other studies, the potential presence of soft-tissue artifact must be acknowledged, even though all

546 experimental procedures were performed carefully to minimize errors from this source. The data

547 were collected while the subjects ran on an instrumented treadmill which certainly was not the

548 first choice of practice environment for most of runners in this study (see metadata file).

549 Therefore, the adopted testing procedures may not be representative of the training and race

550 conditions experienced by the runners and caution should be taken when generalizing from the

551 present findings. In particular, the foot strike index obtained on the treadmill may not necessarily

552 be the same as in overground condition. Nevertheless, the treadmill offers the possibility of

553 controlling gait speed while collecting sufficient trials (footfalls) to represent each subject's

554 pattern. Finally, the subjects wore standard neutral shoes rather than their own shoes. Whether

555 this is an issue is unknown, however we acknowledge that by introducing "new" shoes may

556 require longer familiarization time than what was allowed for the subjects.

557 Despite the fact that the present data set have many applications in future studies, the

558 extent of its use is limited by some factors. Although standardized and detailed described within

559 the manuscript, the data collection procedures may differ from other laboratories with respect,

560 including but not limited to the marker set protocol, the running shoes, the selected gait speed, 
561 the treadmill condition. Hence, caution should be taken when combining this data, particularly

562 when comparing the present data set with others. In addition a Visual 3D biomechanics model

563 (mdh file) is supplied and it can be reused or reproduced in other data sets as long as the same

564 marker set protocol is used. With regards to the treadmill condition, as discussed earlier, caution

565 should be taken when comparing the results with sets of data using different conditions (i.e.

566 overground) or even with different treadmill models. Finally, there is an emerging field of

567 research on wearable sensors to monitor daily life activities including gait that must be

568 acknowledged (Picerno 2017). Whilst the validity and reliability of this technology are not

569 comparable to the data, particularly for non-sagittal movement, obtained in biomechanics

570 laboratories using motion capture systems and force plates, the use of these sensors enhance the

571 ecological validity of the findings since they allow the individuals to run freely in their natural

572 environment and training conditions.

573 The raw dataset provided by this study allows the reuse of this set to test novel

574 approaches to address some of the present limitations. Although a great deal of effort was made

575 to collect and prepare the present dataset, it likely contains deviations, as would any dataset.

576 Therefore, caution should be taken when interpreting the results derived from these data.

577

5785 Conclusions

579 A public dataset of running biomechanics and other data pertaining to running practice

580 has been presented and is available in a public repository. The detailed description of the

581 experimental procedures and the supplied files used for data processing will allow other research

582 groups to generate similar sets of data to expand the current one as well as to reuse them. A

583 number of applications of this dataset can be anticipated, including testing new methods of 
584 reducing data and selecting variables; for educational purposes, and answering specific research

585 questions. With the inclusion of additional subjects, this data set may also serve as reference

586 normative data. In fact, this dataset was useful for addressing the question of whether running

587 speed affects gait biomechanics. The study observed an overall effect of running speed on the

588 kinematic and kinetic variables associated with injuries. In contrast, contrary to our hypothesis,

589 the foot-strike pattern remained unaltered and the eversion angle of the foot was altered only

590 during extreme running speeds. Given the emerging interest in data sharing, there is a need to

591 elaborate standards to present and disseminate gait biomechanics data outlining, among other

592 factors, the minimum set of data required for studying running biomechanics and the potential

593 inclusion of data from wearable sensors.

594

595 References

596 Breine B, Malcolm P, Frederick EC, and De Clercq D. 2014. Relationship between running $597 \quad$ speed and initial foot contact patterns. Med Sci Sports Exerc 46:1595-1603.

$598 \quad 10.1249 /$ MSS.0000000000000267

599 Cappozzo A, Cappello A, Della Croce U, and Pensalfini F. 1997. Surface-marker cluster design 600 criteria for 3-D bone movement reconstruction. IEEE Trans Biomed Eng 44:1165-1174.

601 Cappozzo A, Catani F, Croce UD, and Leardini A. 1995. Position and orientation in space of 602 bones during movement: anatomical frame definition and determination. Clinical 603 Biomechanics (Bristol, Avon) 10:171-178. 10.1016/0268-0033(95)91394-T10

604 Cavanagh PR, and Lafortune MA. 1980. Ground reaction forces in distance running. J Biomech 605 $13: 397-406$. 
606 Cheung RT, Wong RY, Chung TK, Choi RT, Leung WW, and Shek DH. 2016. Relationship

607 between foot strike pattern, running speed, and footwear condition in recreational

608 distance runners. Sports Biomech:1-10. 10.1080/14763141.2016.1226381

609 Daoud AI, Geissler GJ, Wang F, Saretsky J, Daoud YA, and Lieberman DE. 2012. Foot strike

610 and injury rates in endurance runners: a retrospective study. Med Sci Sports Exerc

611 44:1325-1334. 10.1249/MSS.0b013e3182465115

612 Dorn TW, Schache AG, and Pandy MG. 2012. Muscular strategy shift in human running:

613 dependence of running speed on hip and ankle muscle performance. J Exp Biol 215:1944-

$614 \quad$ 1956. $10.1242 /$ jeb.064527

615 Ferber R, Osis ST, Hicks JL, and Delp SL. 2016. Gait biomechanics in the era of data science. $J$ $616 \quad$ Biomech. 10.1016/j.jbiomech.2016.10.033

617 Fukuchi RK, and Duarte M. 2008. Comparison of three-dimensional lower extremity running 618 kinematics of young adult and elderly runners. Journal of Sports Sciences 26:1447-1454.

619904167848 [pii]

$62010.1080 / 02640410802209018$

621 Fukuchi RK, Eskofier BM, Duarte M, and Ferber R. 2011. Support vector machines for detecting 622 age-related changes in running kinematics. Journal of Biomechanics 44:540-542. S0021$6239290(10) 00547-6[\mathrm{pii}]$

$624 \quad 10.1016 /$ j.jbiomech.2010.09.031

625 Fukuchi RK, Stefanyshyn DJ, Stirling L, Duarte M, and Ferber R. 2014. Flexibility, muscle 626 strength and running biomechanical adaptations in older runners. Clinical Biomechanics 627 (Bristol, Avon) 29:304-310. 10.1016/j.clinbiomech.2013.12.007

628 S0268-0033(13)00310-0 [pii] 
629 Fukuchi RK, Stefanyshyn DJ, Stirling L, and Ferber R. 2016. Effects of strengthening and 630 stretching exercise programmes on kinematics and kinetics of running in older adults: a randomised controlled trial. J Sports Sci 34:1774-1781. 10.1080/02640414.2015.1137343

632 Hall JP, Barton C, Jones PR, and Morrissey D. 2013. The biomechanical differences between 633 barefoot and shod distance running: a systematic review and preliminary meta-analysis.

635 Hamner SR, and Delp SL. 2013. Muscle contributions to fore-aft and vertical body mass center 636 accelerations over a range of running speeds. $J$ Biomech 46:780-787.

637 10.1016/j.jbiomech.2012.11.024

638 Hatala KG, Dingwall HL, Wunderlich RE, and Richmond BG. 2013. Variation in foot strike 639 patterns during running among habitually barefoot populations. PLoS One 8:e52548.

640 10.1371/journal.pone.0052548

641 Hespanhol Junior LC, van Mechelen W, Postuma E, and Verhagen E. 2016. Health and 642 economic burden of running-related injuries in runners training for an event: A prospective cohort study. Scand J Med Sci Sports 26:1091-1099. 10.1111/sms.12541

644 Hreljac A. 2004. Impact and overuse injuries in runners. Medicine \& Science in Sports \& 645 Exercise 36:845-849. 00005768-200405000-00017 [pii]

646 Kristianslund E, Krosshaug T, and van den Bogert AJ. 2012. Effect of low pass filtering on joint 647 moments from inverse dynamics: Implications for injury prevention. J Biomech 45:666648 671. S0021-9290(11)00779-2 [pii]

649 10.1016/j.jbiomech.2011.12.011 
650 Lai DT, Begg RK, and Palaniswami M. 2009. Computational intelligence in gait research: a

651 perspective on current applications and future challenges. IEEE Trans Inf Technol

652 Biomed 13:687-702. 10.1109/TITB.2009.2022913

653 Moore IS. 2016. Is There an Economical Running Technique? A Review of Modifiable

654 Biomechanical Factors Affecting Running Economy. Sports Med 46:793-807.

$655 \quad 10.1007 / \mathrm{s} 40279-016-0474-4$

656 Moore JK, Hnat SK, and van den Bogert AJ. 2015. An elaborate data set on human gait and the 657 effect of mechanical perturbations. PeerJ 3:e918. 10.7717/peerj.918

658 Munoz-Jimenez M, Latorre-Roman PA, Soto-Hermoso VM, and Garcia-Pinillos F. 2015.

659 Influence of shod/unshod condition and running speed on foot-strike patterns,

660 inversion/eversion, and vertical foot rotation in endurance runners. J Sports Sci 33:2035-

661 2042. $10.1080 / 02640414.2015 .1026377$

662 Nielsen RO, Nohr EA, Rasmussen S, and Sorensen H. 2013. Classifying running-related injuries 663 based upon etiology, with emphasis on volume and pace. Int J Sports Phys Ther 8:172664179.

665 Nigg BM, Baltich J, Hoerzer S, and Enders H. 2015. Running shoes and running injuries:

666 mythbusting and a proposal for two new paradigms: 'preferred movement path' and

667 'comfort filter'. Br J Sports Med 49:1290-1294. 10.1136/bjsports-2015-095054

668 Petersen J, Nielsen RO, Rasmussen S, and Sorensen H. 2014. Comparisons of increases in knee 669 and ankle joint moments following an increase in running speed from 8 to 12 to $16 \mathrm{~km} . \mathrm{h}(-$

670 1.). Clin Biomech (Bristol, Avon) 29:959-964. 10.1016/j.clinbiomech.2014.09.003 
671 Picerno P. 2017. 25 years of lower limb joint kinematics by using inertial and magnetic sensors:

672 A review of methodological approaches. Gait Posture 51:239-246.

$673 \quad$ 10.1016/j.gaitpost.2016.11.008

674 Santos DA, and Duarte M. 2016. A public data set of human balance evaluations. PeerJ 4:e2648.

$675 \quad 10.7717 /$ peerj. 2648

676 Schache AG, and Baker R. 2007. On the expression of joint moments during gait. Gait Posture $677 \quad 25: 440-452.10 .1016 /$ j.gaitpost.2006.05.018

678 Schache AG, Blanch PD, Dorn TW, Brown NA, Rosemond D, and Pandy MG. 2011. Effect of 679 running speed on lower limb joint kinetics. Med Sci Sports Exerc 43:1260-1271.

680 10.1249/MSS.0b013e3182084929

681

682

683

684

685

686

687

688

689 strategies for increasing running speed. J Orthop Sports Phys Ther 44:813-824. $10.2519 /$ jospt.2014.5433

Stamatakis E, and Chaudhury M. 2008. Temporal trends in adults' sports participation patterns in England between 1997 and 2006: the Health Survey for England. Br J Sports Med 42:901-908. 10.1136/bjsm.2008.048082

Wu G, and Cavanagh PR. 1995. ISB recommendations for standardization in the reporting of kinematic data. $J$ Biomech 28:1257-1261. 
690 Table 1. Details of the 48 anatomical (A) and technical (T) reflective markers used to determine

691 the position and orientation of the body segments during treadmill running. The marker labels

692 are consistent with those stored in files in the c3d format and with the headers of the ASCII

693 marker files.

\begin{tabular}{|c|c|c|c|}
\hline \# & Label & Type & Name \\
\hline 1 & R.ASIS & $\mathrm{T} / \mathrm{A}$ & Right Anterior Superior Iliac Spine \\
\hline 2 & L.ASIS & $\mathrm{T} / \mathrm{A}$ & Left Anterior Superior Iliac Spine \\
\hline 3 & R.PSIS & $\mathrm{T} / \mathrm{A}$ & Right Posterior Iliac Spine \\
\hline 4 & L.PSIS & $\mathrm{T} / \mathrm{A}$ & Left Posterior Iliac Spine \\
\hline 5 & R.Iliac.Crest & $\mathrm{T}$ & Right Iliac Crest \\
\hline 6 & L.Iliac.Crest & $\mathrm{T}$ & Left Iliac Crest \\
\hline 7 & R.Thigh.Top.Lateral & $\mathrm{T}$ & Right Thigh Top Lateral Marker \\
\hline 8 & R.Thigh.Bottom.Lateral & $\mathrm{T}$ & Right Thigh Bottom Lateral Marker \\
\hline 9 & R.Thigh.Top.Medial & $\mathrm{T}$ & Right Thigh Top Medial Marker \\
\hline 10 & R.Thigh.Bottom.Medial & $\mathrm{T}$ & Right Thigh Bottom Medial Marker \\
\hline 11 & R.Shank.Top.Lateral & $\mathrm{T}$ & Right Shank Top Lateral Marker \\
\hline 12 & R.Shank.Bottom.Lateral & $\mathrm{T}$ & Right Shank Bottom Lateral Marker \\
\hline 13 & R.Shank.Top.Medial & $\mathrm{T}$ & Right Shank Top Medial Marker \\
\hline 14 & R.Shank.Bottom.Medial & $\mathrm{T}$ & Right Shank Bottom Medial Marker \\
\hline 15 & R.Heel.Top & $\mathrm{T} / \mathrm{A}$ & Right Heel Top \\
\hline 16 & R.Heel.Bottom & $\mathrm{T} / \mathrm{A}$ & Right Heel Bottom \\
\hline 17 & R.Heel.Lateral & $\mathrm{T}$ & Right Heel Lateral \\
\hline
\end{tabular}




\begin{tabular}{|c|c|c|c|}
\hline 18 & L.Thigh.Top.Lateral & $\mathrm{T}$ & Left Thigh Top Lateral Marker \\
\hline 19 & L.Thigh.Bottom.Lateral & $\mathrm{T}$ & Left Thigh Bottom Lateral Marker \\
\hline 20 & L.Thigh.Top.Medial & $\mathrm{T}$ & Left Thigh Top Medial Marker \\
\hline 21 & L.Thigh.Bottom.Medial & $\mathrm{T}$ & Left Thigh Bottom Medial Marker \\
\hline 22 & L.Shank.Top.Lateral & $\mathrm{T}$ & Left Shank Top Lateral Marker \\
\hline 23 & L.Shank.Bottom.Lateral & $\mathrm{T}$ & Left Shank Bottom Lateral Marker \\
\hline 24 & L.Shank.Top.Medial & $\mathrm{T}$ & Left Shank Top Medial Marker \\
\hline 25 & L.Shank.Bottom.Medial & $\mathrm{T}$ & Left Shank Bottom Medial Marker \\
\hline 26 & L.Heel.Top & $\mathrm{T} / \mathrm{A}$ & Left Heel Top \\
\hline 27 & L.Heel.Bottom & $\mathrm{T} / \mathrm{A}$ & Left Heel Bottom \\
\hline 28 & L.Heel.Lateral & $\mathrm{T}$ & Left Heel Lateral \\
\hline 29 & R.GTR & A & Right Greater Trochanter \\
\hline 30 & R.Knee & A & Right Knee \\
\hline 31 & R.Knee.Medial & A & Right Knee Medial \\
\hline 32 & R.HF & A & Right Head of Fibula \\
\hline 33 & R.TT & A & Right Tibial Tuberosity \\
\hline 34 & R.Ankle & A & Right Ankle \\
\hline 35 & R.Ankle.Medial & A & Right Ankle Medial \\
\hline 36 & R.MT1 & A & Right 1st Metatarsal \\
\hline 37 & R.MT5 & A & Right 5th Metatarsal \\
\hline 38 & R.MT2 & A & Right 2nd Metatarsal \\
\hline 39 & L.GTR & A & Left Greater Trochanter \\
\hline 40 & L.Knee & A & Left Knee \\
\hline
\end{tabular}




\begin{tabular}{llcl}
\hline 41 & L.Knee.Medial & A & Left Knee Medial \\
\hline 42 & L.HF & A & Left Head of Fibula \\
\hline 43 & L.TT & A & Left Tibial Tuberosity \\
\hline 44 & L.Ankle & A & Left Ankle \\
\hline 45 & L.Ankle.Medial & A & Left Ankle Medial \\
\hline 46 & L.MT1 & A & Left 1st Metatarsal \\
\hline 47 & L.MT5 & A & Left 5th Metatarsal \\
\hline 48 & L.MT2 & A & Left 2nd Metatarsal
\end{tabular}


695 Table 2. Description of the 12 file names per subject in the Running Biomechanics Data set.
Type
File name
Description

\begin{tabular}{lll}
\hline C3D & RBDSxxstatic.c3d & Standing calibration trial data \\
\hline C3D & RBDSxxrunT25.c3d & Markers and forces data for running at $2.5 \mathrm{~m} / \mathrm{s}$ \\
\hline C3D & RBDSxxrunT35.c3d & Markers and forces data for running at $3.5 \mathrm{~m} / \mathrm{s}$ \\
\hline C3D & RBDSxxrunT45.c3d & Markers and forces data for running at $4.5 \mathrm{~m} / \mathrm{s}$ \\
\hline ASCII & RBDSxxstatic.txt & Standing calibration trial data \\
\hline
\end{tabular}

ASCII RBDSxxrunT25markers.txt Markers data for running at $2.5 \mathrm{~m} / \mathrm{s}$

ASCII RBDSxxrunT35markers.txt Markers data for running at $3.5 \mathrm{~m} / \mathrm{s}$

ASCII RBDSxxrunT45markers.txt Markers data for running at $4.5 \mathrm{~m} / \mathrm{s}$

ASCII RBDSxxrunT25forces.txt Forces data for running at $2.5 \mathrm{~m} / \mathrm{s}$

ASCII RBDSxxrunT35forces.txt Forces data for running at $3.5 \mathrm{~m} / \mathrm{s}$

ASCII RBDSxxrunT45forces.txt Forces data for running at $4.5 \mathrm{~m} / \mathrm{s}$

ASCII RBDSxxprocessed.txt Time-normalized kinematics and kinetics data for all

speed conditions

696 
697 Table 3. Arrangement of processed data in the first 25 columns, comprising the joint angles, joint torques, GRFs, and joint powers for 698 one speed condition and one lower limb.

699

\begin{tabular}{|c|c|c|c|c|c|c|c|c|c|c|c|c|c|c|c|c|c|c|c|c|c|c|c|}
\hline \multirow{3}{*}{ Cycle $[\%]$} & \multicolumn{8}{|c|}{ Joint angle $\left[^{\circ}\right]$} & \multicolumn{9}{|c|}{ Joint torque $[\mathrm{Nm} / \mathrm{kg}]$} & \multirow{2}{*}{\multicolumn{3}{|c|}{ GRF $[\mathrm{N} / \mathrm{kg}]$}} & \multicolumn{3}{|c|}{ Joint power [W/kg] } \\
\hline & \multicolumn{3}{|c|}{ HIP } & \multicolumn{2}{|c|}{ KNEE } & \multicolumn{3}{|c|}{ ANKLE } & \multicolumn{3}{|c|}{ HIP } & \multicolumn{3}{|c|}{ KNEE } & \multicolumn{3}{|c|}{ ANKLE } & & & & \multirow{2}{*}{ HIP } & \multirow{2}{*}{ KNEE } & \multirow{2}{*}{ ANKLE } \\
\hline & $\mathrm{X}$ & $\mathrm{Y}$ & $\mathrm{Z} \quad \mathrm{X}$ & $\mathrm{Y}$ & $\mathrm{Z}$ & $\mathrm{X}$ & $\mathrm{Y}$ & $\mathrm{Z}$ & $\mathrm{X}$ & $\mathrm{Y}$ & $\mathrm{Z}$ & $\mathrm{X}$ & $\mathrm{Y}$ & $\mathrm{Z}$ & $\mathrm{X}$ & $\mathrm{Y}$ & $\mathrm{Z}$ & $\mathrm{X}$ & $\mathrm{Y}$ & $\mathrm{Z}$ & & & \\
\hline 1 & 2 & 3 & 45 & 6 & 7 & 8 & 9 & 10 & 11 & 12 & 13 & 14 & 15 & 16 & 17 & 18 & 19 & 20 & 21 & 22 & 23 & 24 & 25 \\
\hline
\end{tabular}

700

701 
Table 4. Descriptive and inferential statistics for the kinematic and kinetic variables of 28 subjects during treadmill running at $2.5 \mathrm{~m} / \mathrm{s}$, $3.5 \mathrm{~m} / \mathrm{s}$, and $4.5 \mathrm{~m} / \mathrm{s}$. In the results of the post-hoc multiple comparisons with Bonferroni adjustments, 0 indicates no difference, and 1 indicates a significant difference in the pairwise comparison. The symbol \# indicate variables compared using the Kruskal-Wallis test.

\begin{tabular}{|c|c|c|c|c|c|c|c|c|c|c|}
\hline \multirow[b]{2}{*}{ Variables } & \multirow{2}{*}{$\begin{array}{c}2.5 \mathrm{~m} / \mathrm{s} \\
\text { Mean } \pm \mathrm{SD}\end{array}$} & \multirow{2}{*}{$\begin{array}{c}3.5 \mathrm{~m} / \mathrm{s} \\
\text { Mean } \pm \text { SD }\end{array}$} & \multirow{2}{*}{$\begin{array}{c}4.5 \mathrm{~m} / \mathrm{s} \\
\text { Mean } \pm \text { SD }\end{array}$} & \multicolumn{2}{|c|}{$\begin{array}{c}\text { Mean } \\
\text { relative } \\
\text { difference }\end{array}$} & \multicolumn{2}{|c|}{ ANOVA } & \multicolumn{3}{|c|}{ Multiple comparisons } \\
\hline & & & & $\begin{array}{l}\text { V2- } \\
\text { V1 }\end{array}$ & $\begin{array}{l}\text { V3- } \\
\text { V1 }\end{array}$ & $\begin{array}{c}\text { F or } \\
\chi^{2}\end{array}$ & $\begin{array}{c}\text { p- } \\
\text { value }\end{array}$ & $\begin{array}{c}\mathrm{V} 1 \mathrm{~V} \\
2 '\end{array}$ & $\begin{array}{c}\text { V1V } \\
3 '\end{array}$ & $\begin{array}{c}\mathrm{V} 2 \mathrm{~V} \\
3 '\end{array}$ \\
\hline Stride Length [m] & $1.86 \pm 0.11$ & $2.46 \pm 0.15$ & $2.96 \pm 0.20$ & 0.60 & 1.10 & $\begin{array}{r}335.3 \\
9 \#\end{array}$ & 0.000 & 1 & 1 & 1 \\
\hline Cadence [strides per minute] & $\begin{array}{r}80.82 \pm \\
4.63\end{array}$ & $85.68 \pm 5.27$ & $91.74 \pm 6.69$ & 4.86 & 10.92 & 26.72 & 0.000 & 1 & 1 & 1 \\
\hline Stride Width [m] & $0.10 \pm 0.02$ & $0.09 \pm 0.02$ & $0.08 \pm 0.02$ & -0.01 & -0.01 & 2.60 & 0.080 & - & - & - \\
\hline Max Hip Flx Angle $\left[^{\circ}\right]$ & $\begin{array}{r}43.75 \pm \\
6.06 \\
\end{array}$ & $52.76 \pm 5.75$ & $60.50 \pm 6.06$ & 9.01 & 16.76 & 55.48 & 0.000 & 1 & 1 & 1 \\
\hline Max Hip Ext Angle $\left[^{\circ}\right]$ & $\begin{array}{r}-3.58 \pm \\
4.85 \\
\end{array}$ & $-7.95 \pm 4.58$ & $\begin{array}{r}-11.75 \pm \\
4.78 \\
\end{array}$ & 4.37 & 8.18 & 20.90 & 0.000 & 1 & 1 & 1 \\
\hline Max Knee Flx Angle ${ }^{\circ}{ }^{\circ}$ & $\begin{array}{r}93.52 \pm \\
10.36 \\
\end{array}$ & $\begin{array}{r}108.68 \pm \\
10.65 \\
\end{array}$ & $\begin{array}{r}119.12 \pm \\
10.37 \\
\end{array}$ & 15.15 & 25.59 & 42.37 & 0.000 & 1 & 1 & 1 \\
\hline Knee ABD Impulse [Nms] & $0.20 \pm 0.06$ & $0.20 \pm 0.06$ & $0.20 \pm 0.06$ & 0.00 & 0.01 & 0.10 & 0.905 & - & - & - \\
\hline Max Ankle DF Angle $\left[^{\circ}\right]$ & $\begin{array}{r}26.36 \pm \\
2.93 \\
\end{array}$ & $26.54 \pm 2.49$ & $26.79 \pm 2.51$ & 0.18 & 0.43 & 0.19 & 0.831 & - & - & - \\
\hline Max Ankle PF Angle $\left.{ }^{\circ}\right]^{\circ}$ & $\begin{array}{r}-16.62 \pm \\
5.50\end{array}$ & $\begin{array}{r}-20.47 \pm \\
4.71\end{array}$ & $\begin{array}{r}-23.17 \pm \\
4.72\end{array}$ & 3.84 & 6.54 & 12.15 & 0.000 & 1 & 1 & 0 \\
\hline Max Eversion Angle $\left[^{\circ}\right]$ & $\begin{array}{r}-4.91 \pm \\
2.74\end{array}$ & $-6.59 \pm 2.99$ & $-7.81 \pm 3.59$ & 1.68 & 2.90 & 6.07 & 0.004 & 0 & 1 & 0 \\
\hline Max Hip Flx Torque [Nm/kg] & $0.78 \pm 0.11$ & $1.15 \pm 0.14$ & $1.49 \pm 0.19$ & 0.37 & 0.70 & $\begin{array}{r}150.4 \\
2^{\#}\end{array}$ & 0.000 & 1 & 1 & 1 \\
\hline Max Hip Ext Torque [Nm/kg] & $\begin{array}{r}-1.06 \pm \\
0.14 \\
\end{array}$ & $-1.37 \pm 0.19$ & $-1.67 \pm 0.21$ & 0.31 & 0.61 & 76.50 & 0.000 & 1 & 1 & 1 \\
\hline Max Knee Ext Torque & $2.84 \pm 0.45$ & $3.18 \pm 0.50$ & $3.41 \pm 0.47$ & 0.34 & 0.57 & 10.28 & 0.000 & 1 & 1 & 0 \\
\hline
\end{tabular}




\begin{tabular}{|c|c|c|c|c|c|c|c|c|c|c|}
\hline$[\mathrm{Nm} / \mathrm{kg}]$ & & & & & & & & & & \\
\hline $\begin{array}{l}\text { Max Ankle PF Torque } \\
{[\mathrm{Nm} / \mathrm{kg}]}\end{array}$ & $2.03 \pm 0.22$ & $2.23 \pm 0.23$ & $2.34 \pm 0.25$ & 0.21 & 0.31 & 13.11 & 0.000 & 1 & 1 & 0 \\
\hline $\begin{array}{l}\text { Max Ankle DF Torque } \\
{[\mathrm{Nm} / \mathrm{kg}]}\end{array}$ & $\begin{array}{r}-0.14 \pm \\
0.11 \\
\end{array}$ & $-0.23 \pm 0.16$ & $-0.32 \pm 0.20$ & 0.09 & 0.18 & $8.61^{\#}$ & 0.000 & 0 & 1 & 0 \\
\hline Hip Pos Work $[\mathrm{J} / \mathrm{kg}]$ & $0.80 \pm 0.20$ & $1.49 \pm 0.30$ & $2.43 \pm 0.39$ & 0.69 & 1.62 & $\begin{array}{r}195.1 \\
6^{\#}\end{array}$ & 0.000 & 1 & 1 & 1 \\
\hline Hip Neg Work $[\mathrm{J} / \mathrm{kg}]$ & $\begin{array}{r}-0.27 \pm \\
0.09 \\
\end{array}$ & $-0.42 \pm 0.12$ & $-0.66 \pm 0.22$ & 0.16 & 0.39 & $44.24^{\#}$ & 0.000 & 1 & 1 & 1 \\
\hline Knee Pos Work $[\mathrm{J} / \mathrm{kg}]$ & $0.69 \pm 0.17$ & $0.86 \pm 0.19$ & $0.92 \pm 0.23$ & 0.17 & 0.23 & 10.08 & 0.000 & 1 & 1 & 0 \\
\hline Knee Neg Work [J/kg] & $\begin{array}{r}-1.50 \pm \\
0.18 \\
\end{array}$ & $-2.15 \pm 0.24$ & $-3.03 \pm 0.30$ & 0.65 & 1.53 & $\begin{array}{r}274.0 \\
7 \#\end{array}$ & 0.000 & 1 & 1 & 1 \\
\hline Ankle Pos Work [J/kg] & $0.64 \pm 0.10$ & $0.78 \pm 0.10$ & $0.95 \pm 0.15$ & 0.14 & 0.32 & 50.90 & 0.000 & 1 & 1 & 1 \\
\hline Ankle Neg Work [J/kg] & $\begin{array}{r}-0.58 \pm \\
0.13\end{array}$ & $-0.77 \pm 0.14$ & $-0.96 \pm 0.15$ & 0.19 & 0.39 & 52.86 & 0.000 & 1 & 1 & 1 \\
\hline $\begin{array}{l}\text { GRF Brak Impulse A-P } \\
{[\mathrm{Ns} / \mathrm{kg}]}\end{array}$ & $\begin{array}{r}-0.34 \pm \\
0.10 \\
\end{array}$ & $-0.49 \pm 0.13$ & $-0.56 \pm 0.14$ & 0.15 & 0.22 & 22.16 & 0.000 & 1 & 1 & 0 \\
\hline $\begin{array}{l}\text { GRF Prop Impulse A-P } \\
{[\mathrm{Ns} / \mathrm{kg}]}\end{array}$ & $0.27 \pm 0.07$ & $0.33 \pm 0.09$ & $0.40 \pm 0.12$ & 0.07 & 0.13 & 12.75 & 0.000 & 1 & 1 & 1 \\
\hline $\begin{array}{l}\text { GRF Pos Impulse Vertical } \\
\text { [Ns/kg] }\end{array}$ & $5.04 \pm 0.09$ & $5.12 \pm 0.10$ & $5.19 \pm 0.10$ & 0.08 & 0.15 & 17.44 & 0.000 & 1 & 1 & 1 \\
\hline
\end{tabular}


709 Figure 1

710 Overview of the Laboratory of Biomechanics and Motor Control

711 Figure 1. Expanded view of the Laboratory of Biomechanics and Motor Control (BMClab),

712 showing 10 of the 12 motion-capture system cameras (marked with red circles), the instrumented

713 treadmill, and the laboratory-coordinate system.

714

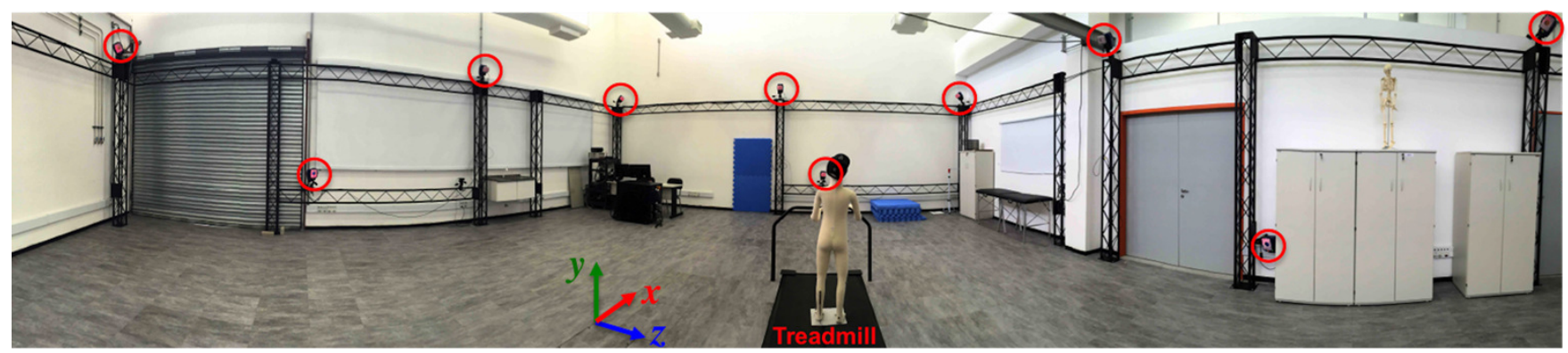

715

716 
717 Figure 2

718 Marker set protocol

719 Figure 2. The technical and anatomical marker set protocol during an anatomical calibration trial

720 in the anterior (A), lateral (B) and posterior (C) views.

721

722

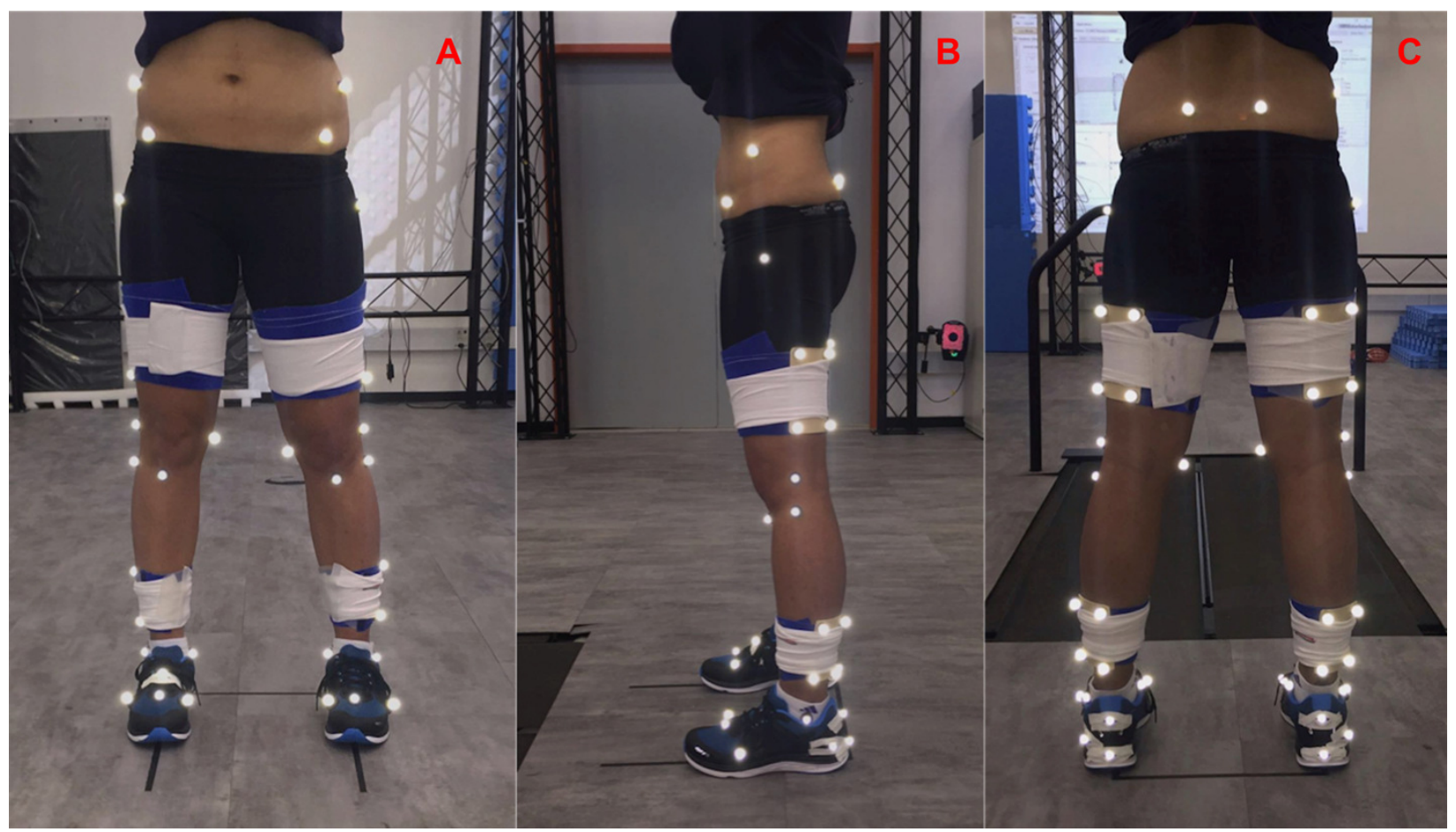


724 Figure 3

725 Lower extremity joint angles

726 Figure 3. Ensemble time series of 3D hip (A, B and C), knee (D, E and F) and ankle (G, H and I)

727 joint angles across participants during treadmill running at $2.5 \mathrm{~m} / \mathrm{s}, 3.5 \mathrm{~m} / \mathrm{s}$, and $4.5 \mathrm{~m} / \mathrm{s}$.

728
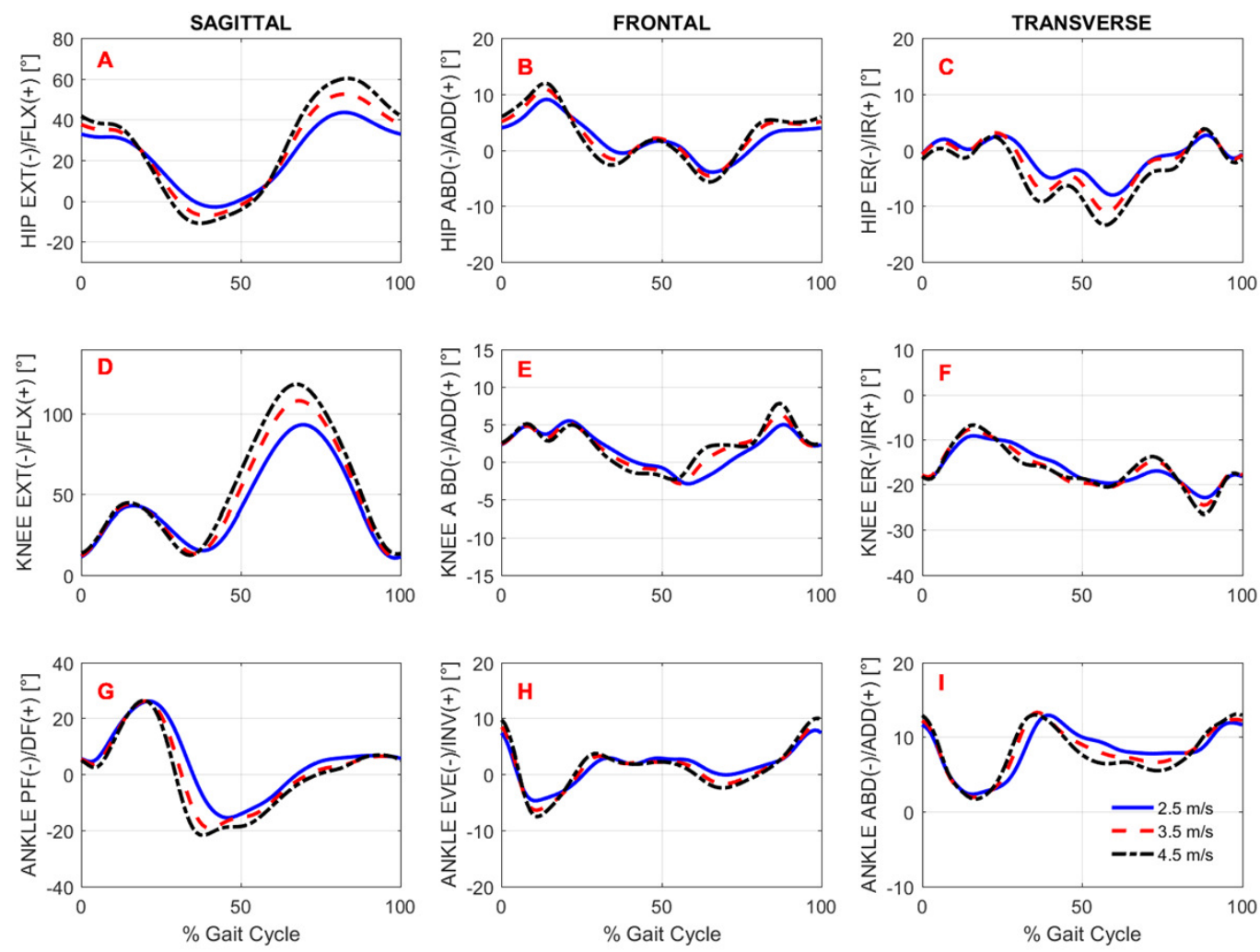

729

730 
731 Figure 4

732 Lower extremity joint torques

733 Figure 4. Ensemble time series of 3D hip (A, B and C), knee (D, E and F) and ankle (G, H and I)

734 joint torques across participants during treadmill running at $2.5 \mathrm{~m} / \mathrm{s}, 3.5 \mathrm{~m} / \mathrm{s}$, and $4.5 \mathrm{~m} / \mathrm{s}$.

735
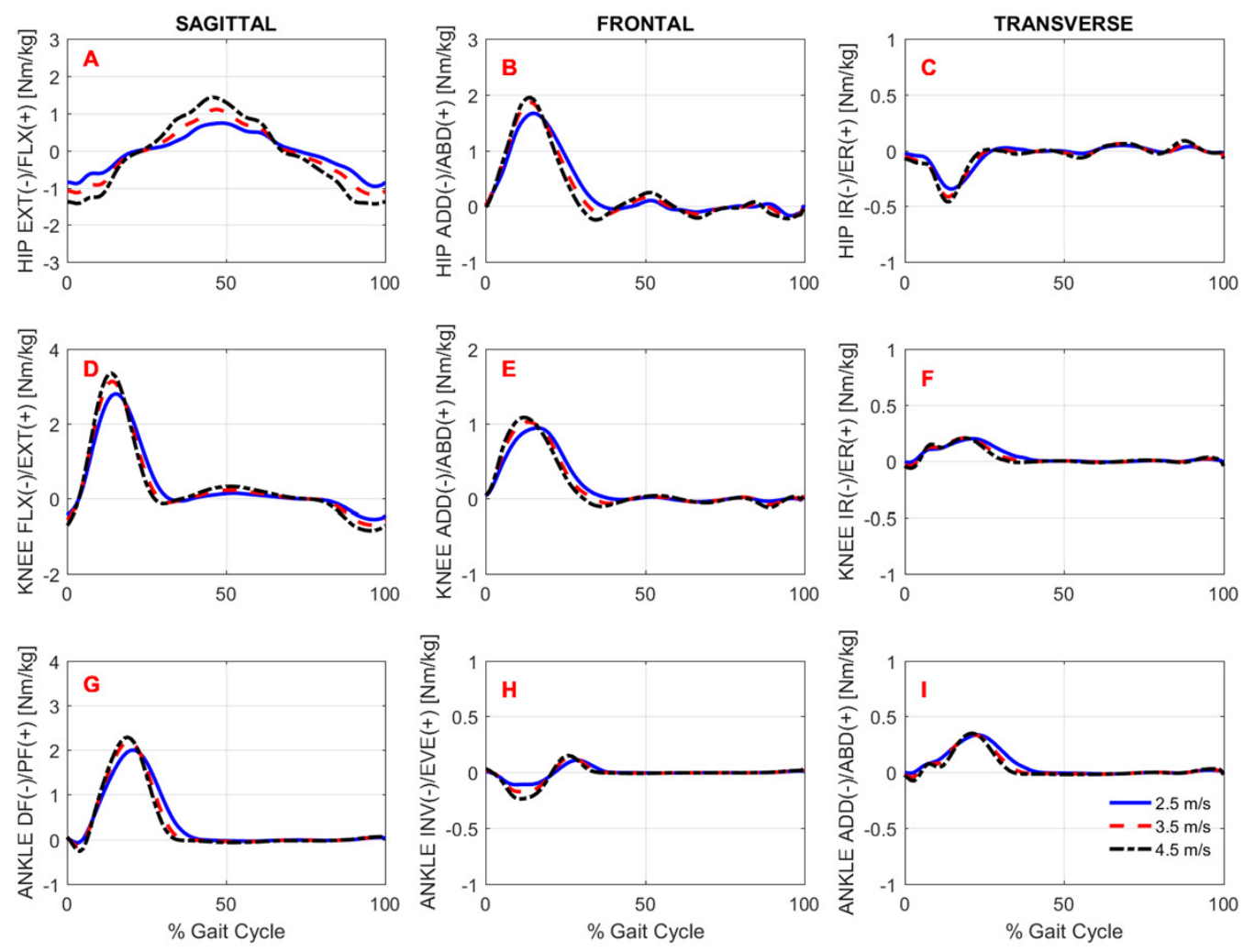

736

737 
738 Figure 5

739 Ground reaction forces and joint powers

740 Figure 5. Ensemble time series of 3D GRF forces (A, B and C) and hip (D), knee (E), and ankle

741 (F) powers at the sagittal plane across participants during treadmill running at $2.5 \mathrm{~m} / \mathrm{s}, 3.5 \mathrm{~m} / \mathrm{s}$, 742 and $4.5 \mathrm{~m} / \mathrm{s}$.

743
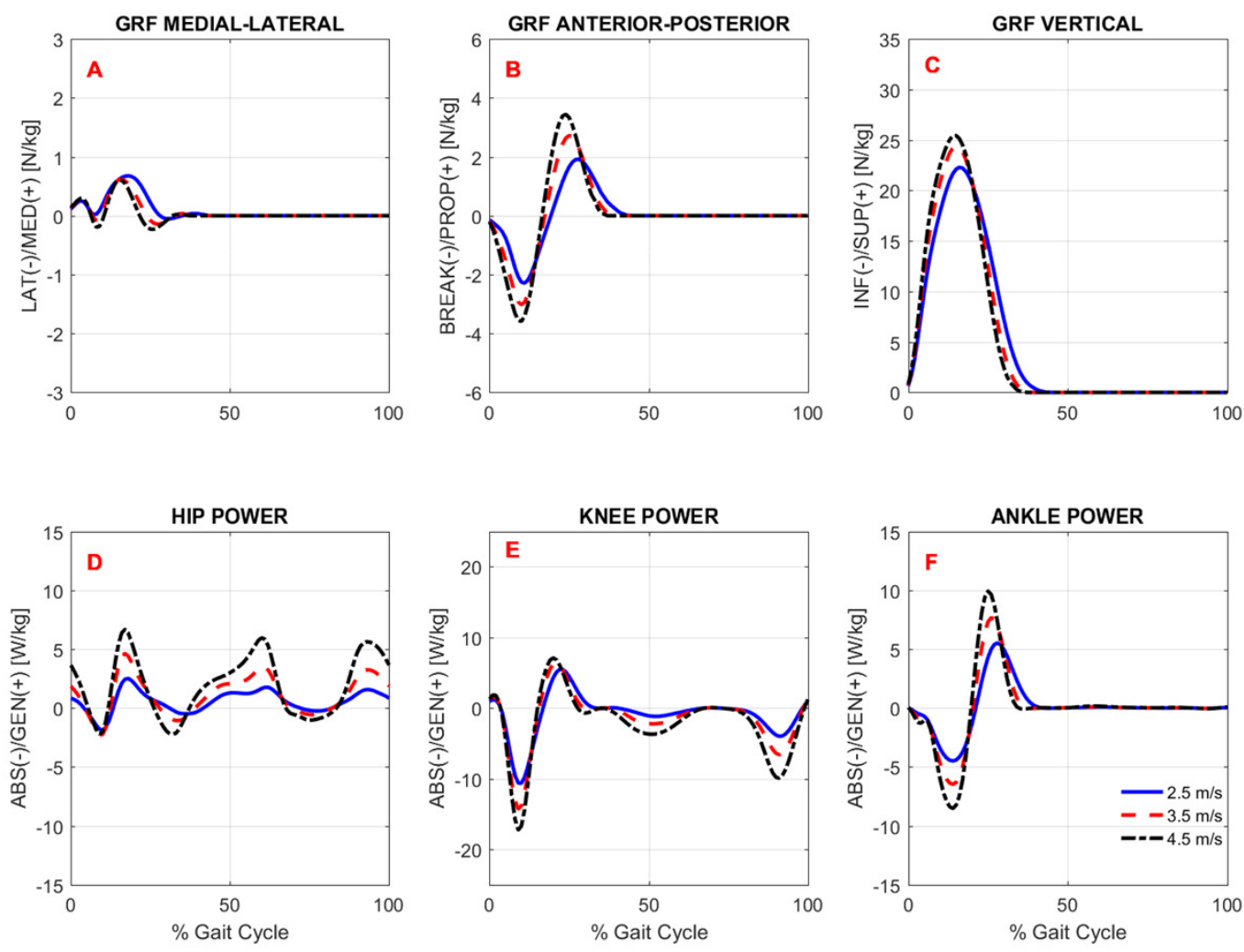

744

745 
746 Figure 6

747 Kinematic and kinetic values distribution across the range of running speeds.

748 Figure 6. Plots highlighting the distribution of the 28 subjects' values across running speeds in

749 the kinematic (A to $\mathrm{G}$ ) and kinetic ( $\mathrm{H}$ to $\mathrm{U}$ ) variables. Significant differences in the post-hoc

750 analyses are indicated by the symbols $*,+$, and $\Delta . *$ Significant difference in all pairwise

751 comparisons. ${ }^{+}$Significant difference between $2.5 \mathrm{~m} / \mathrm{s}$ vs. $3.5 \mathrm{~m} / \mathrm{s}$ and between $2.5 \mathrm{~m} / \mathrm{s}$ vs. 4.5

$752 \mathrm{~m} / \mathrm{s} .{ }_{\Delta}^{\Delta}$ Significant difference only between $2.5 \mathrm{~m} / \mathrm{s}$ vs. $4.5 \mathrm{~m} / \mathrm{s}$.
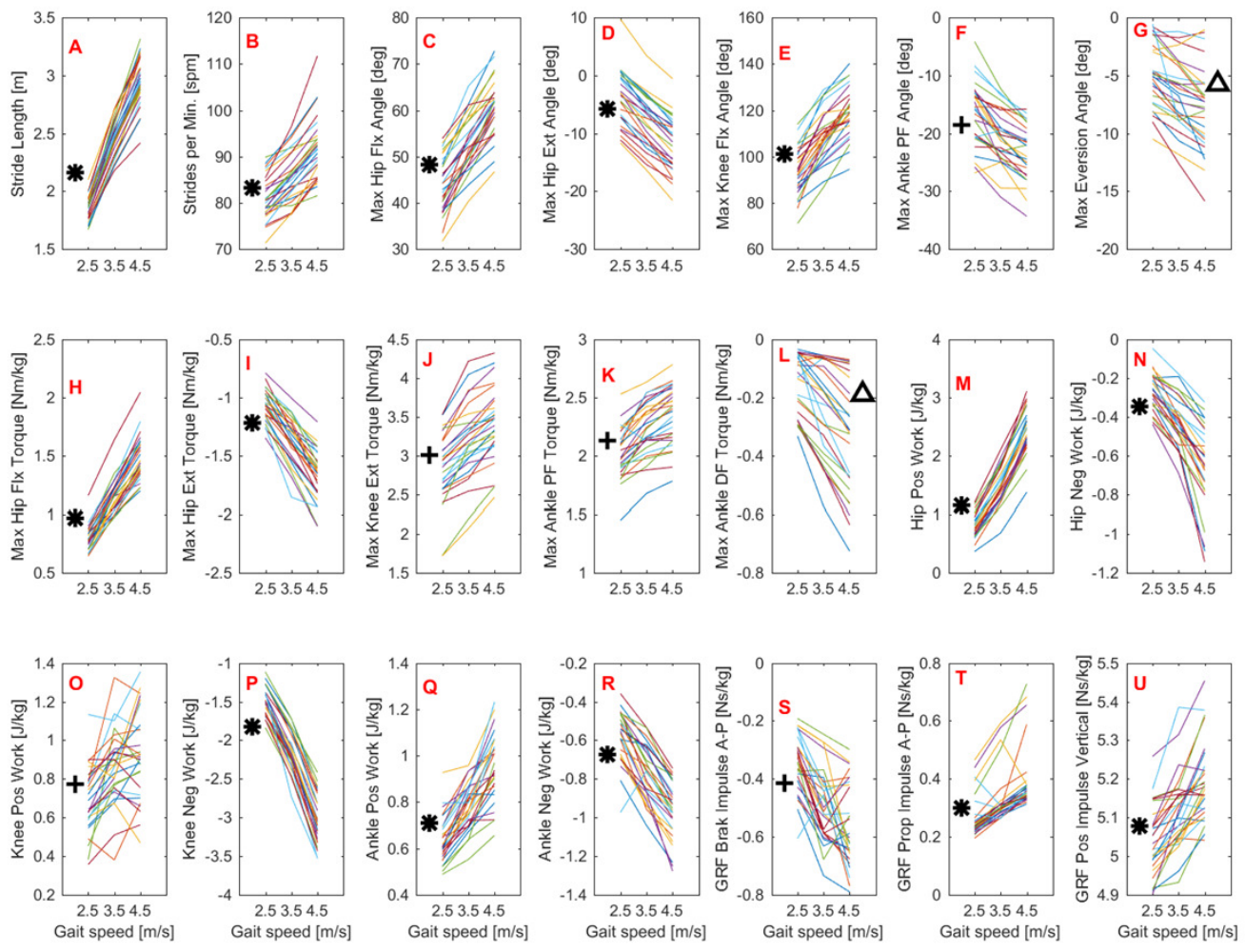

754 